\title{
Preliminary design studies of an extraction of the USR
}

\author{
P. Schmid • K.-U. Kühnel • A. Papash • C. P. Welsch
}

Published online: 28 August 2009

(C) The Author(s) 2009. This article is published with open access at Springerlink.com

\begin{abstract}
The next-generation Facility for Low energy Antiproton and Ion Research (FLAIR) at GSI is going to be a dedicated research facility for ion research in the $\mathrm{keV}$ range. These ion beams will allow to explore fundamental properties in matterantimatter research at ultra-low energies of only $20 \mathrm{keV} / \mathrm{q}$ in hitherto impossible experiments. To provide these very low energy beams, the Ultra-low energy $\underline{\text { Storage }}$ Ring (USR), an electrostatic synchrotron, will play a major role within the FLAIR complex. It combines the electrostatic storage mode with deceleration from an initial energy of $300 \mathrm{keV} / \mathrm{q}$ down to $20 \mathrm{keV} / \mathrm{q}$ - as well as an efficient beam cooling. To fulfill its role as a multi-purpose experimental facility, the design of the USR has not only to cover in-ring experiments, but needs to include a highly flexible beam extraction for serving different external experiments as well.
\end{abstract}

Keywords Electrostatic $\cdot$ Storage ring $\cdot$ Extraction

PACS $41.85 . \mathrm{Ar} \cdot 29.20 . \mathrm{Ba} \cdot 41.85 . \mathrm{Ja}$

P. Schmid · K.-U. Kühnel · A. Papash · C.P. Welsch ( $ه)$

Max Planck Institute for Nuclear Physics, Saupfercheckweg 1, 69117 Heidelberg, Germany

e-mail: carsten.welsch@quasar-group.org

P. Schmid · C.P. Welsch · A. Papash

Gesellschaft für Schwerionenforschung, Planckstrasse 1, 64291 Darmstadt, Germany

C.P. Welsch

The Cockcroft Institute, Warrington, WA4 4AD, UK

C.P. Welsch

Department of Physics, The University of Liverpool, Liverpool, L69 7ZE, UK 


\section{Introduction}

The next-generation antiproton facility at GSI, the Facility for $\underline{\text { Antiproton and Ion }}$ Research (FAIR), will not only provide future users with high fluxes of antiprotons in the high energy range, but will include a dedicated research program with low energy antiprotons in the $\mathrm{keV}$ regime, realized with the FLAIR project [1]. Within this planned accelerator complex the deceleration of antiprotons, arriving from the NESR storage ring housed in the FAIR facility with an initial energy of $30 \mathrm{MeV}$ down to ultralow energies of $20 \mathrm{keV}$ will be realized in two steps. First, the beam is decelerated to an energy of $300 \mathrm{keV}$ in a conventional magnetic ring, the Low

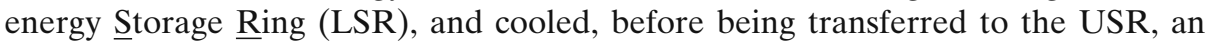
electrostatic synchrotron [2]. In this circular machine the deceleration to a final energy of $20 \mathrm{keV}$ will be realized.

As the ring aims to be a multi-user facility by itself, a high luminosity, low emittance and low momentum spread of the beam together with variable beam shapes are required [3]. External experiments for precision studies, like e.g. trap experiments, will form an integral part of FLAIR and the USR needs to provide fast as well as slow extraction, for the first time in an electrostatic storage ring [4]. A more detailed description of the ring is given in [5].

\section{Preliminary design of a highly flexible extraction scheme}

\subsection{The fast extraction scheme of the USR}

In the fast extraction process, all stored particles are moved in a single turn over the septum wall into the extraction channel. To initiate such a process in the USR, the beam will be moved by a local orbit bump slowly towards the extraction septum, located in one of the straight sections. To allow for the highest flexibility during this process, this bump will be created by four dedicated electrostatic deflectors. This controls not only the bump height, but at the same time, in contrast to a three-kicker bump, creates a parallel beam in the center between the electrodes. Due to the closed orbit bump, the necessary "kick-out" field strengths-used for the actual extraction process - can be reduced.

As soon as the beam moves along the bump, an additional kicking voltage is applied to one of the bump electrodes. This effectively pushes the beam over the septum wall into the extraction channel. A simulation of such a fast extraction process was performed, using the tracking code SIMION [6]. For a $20 \mathrm{keV}$ (anti)proton beam the required electrode potentials are below $\pm 800 \mathrm{~V}$, which can be easily realized.

\subsection{Operation scheme for slow extraction}

In case an external experiment requires a continuous beam of antiprotons, e.g. for measurements of $\frac{d E}{d x}$ and ionization measurements with slow antiprotons or x-ray spectroscopy of $\bar{p}$-light nuclei, as measured at LEAR or the AD [7,8], the only option is the use of slow extraction from the storage ring.

The principle behind slow resonant extraction is the use of controlled transverse resonances in the synchrotron for beam extraction $[9,10]$. One additional advantage 
Fig. 1 Phase-space distribution at the position of the electrostatic septum (top) and a schematic depiction of the RF-knock-out extraction method (bottom)
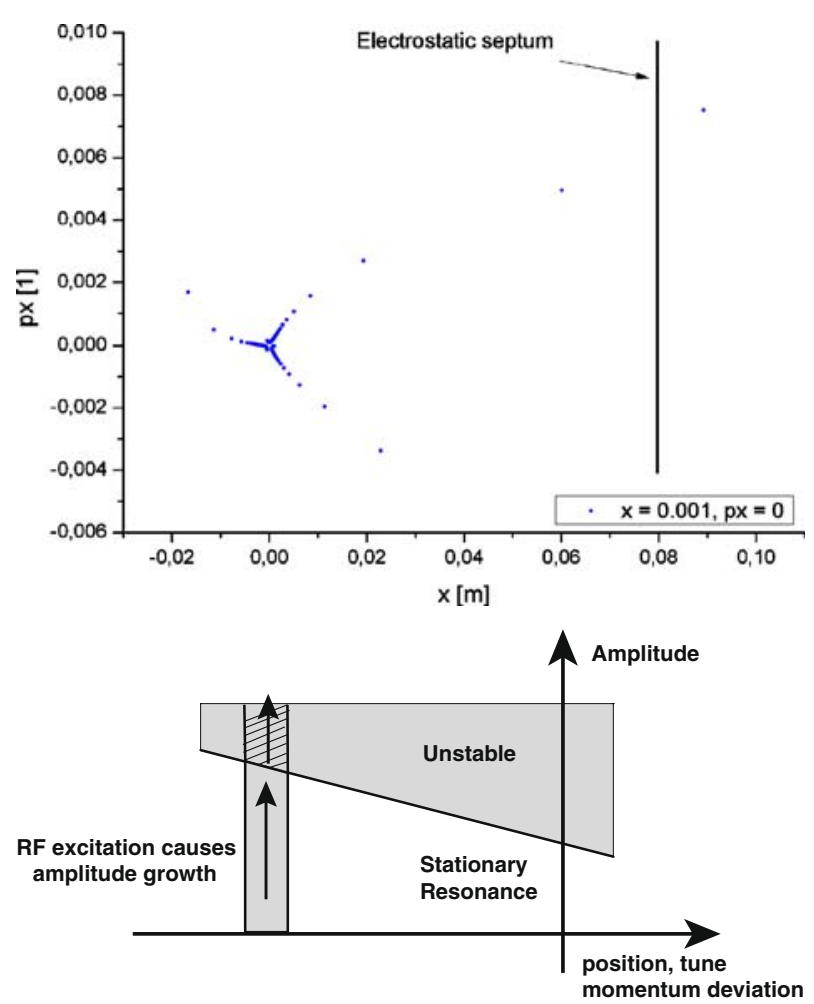

of such a stochastic feeding is that the extracted momentum bite is not only stable but also narrower than in the internal circulating beam. This particular extraction mode is normally realized by the application of a so-called third-order resonance. Thereby the betatron tune in the extraction plane has to be close to the resonance condition, for the case of the said resonance: $3 \cdot \mathrm{Q}=$ integer.

Before starting the extraction process, the circulating beam inside the USR has to be set-up with respect to the resonance. The horizontal tune is moved close to the $3 \cdot \mathrm{Q}=8$ resonance by varying the strength of all quadrupoles around the machine. At the same time the field of the dedicated electrostatic sextupole is raised to its nominal strength $\mathrm{S}$ in a quasi-adiabatic way. This leads to a distortion of the elliptical trajectories (in un-normalized phase-space) and the available phase-space is split into stable and unstable regions by the separatrices that define the stable triangle.

In this process a particle will move on a stable orbit, if its emittance is smaller than or equal to the area of the stability triangle. Otherwise the particle is forced onto one of the separatrix arms (see Fig. 1 as an example), where it spirals outwards, returning to the initial arm at a position further away from the beam center every three turns. The step-size increases non-linearly and is dependent on the sextupole strength $\mathrm{S}$.

It can be assumed that the particles move on a stable orbit after the machine has been set to extraction conditions. Therefore the particles have to be moved from the inner, stable area of the triangle onto the separatrix arms during the course of the extraction. For this step several different mechanisms are currently operational in different synchrotrons worldwide. For the slow extraction from the USR, a stochastic 
extraction scheme, using some transversal RF noise will be applied. In this scheme, the oscillation amplitudes of the particles are increased, moving the individual tune of the particles into the unstable resonance [11]. The advantage of this method is not only the possibility to exactly control the spill period, but more importantly, the overall setting of the lattice can be kept constant during the whole spill time, as shown schematically in the Steinbach diagram for this process, depicted in Fig. 1.

Tracking simulations of such a $3 \cdot \mathrm{Q}=8$ resonant extraction in the USR using the thin-lens tracking option in the numerical simulation code MAD-X [12] were performed, leading to the geometry and the settings of the extraction process. The phase space plot of the beam resulting from these investigations is depicted in Fig. 1.

\subsubsection{Electrostatic sextupole design}

The slow extraction process requires a dedicated electrostatic sextupole to cause the phase-space distortion. Thus, first real-field simulations for a possible sextupole design were performed. A model of a sextupole, including the hyperbolic electrode shape necessary for a homogeneous field distribution, was implemented into TOSCA-3D [13]. This model is presently used to optimize the overall geometry for an effective minimization of the fringe fields. We are aiming at finalizing the design of the whole extraction region until the end of 2009 and to have a prototype installation setup until mid 2010.

Acknowledgements The authors would like to thank Manfred Grieser for the discussions and fruitful cooperation. Work supported by GSI and the Helmholtz Association of National Research Centers (HGF) under contract number VH-NG-328.

Open Access This article is distributed under the terms of the Creative Commons Attribution Noncommercial License which permits any noncommercial use, distribution, and reproduction in any medium, provided the original author(s) and source are credited.

\section{References}

1. Widmann, E., et al.: FLAIR - technical proposal. http:/www.oeaw.ac.at/smi/flair (2005). Accessed 20 Oct 2008

2. Welsch, C.P.: An ultra-low-energy storage ring at FLAIR. Nucl. Instrum. Methods A 546, 405417 (2005)

3. Welsch, C.P., et al.: FLAIR - a facility for low-energy antiproton and ion research. Hyperfine Interact. 172, 71-80 (2006)

4. Schmid, Ph., et al.: Preliminary Design of a highly-flexible extraction scheme for the USR. In: Proc. European Part. Acc. Conf., pp. 3407-3409. Genova, Italy (2008)

5. Welsch, C.P., et al.: Present status of the USR project. Hyperfine Interact. (2009, in press)

6. Dahl, D.A.: SIMION for the personal computer in reflection. Int. J. Mass Spectrom. 200, 3 (2000) (source: Scientific Instrument Services, Inc., Ringoes, NJ, www.simion.com)

7. Knudsen, H., et al.: Ionization of helium and argon by very slow antiproton impact. Phys. Rev. Lett. 101, 043201 (2008)

8. Gotta, D.: Precision spectroscopy of light exotic atoms. Prog. Part. Nucl. Phys. 52, 133-195 (2004)

9. Strolin, P.: Third-order resonance slow extraction from alternating gradient synchrotrons. CERN/ISR/TH/66-40 (1966)

10. Baird, S., et al.: Stochastic cooling and storage tests with oxygen ions in lear. In: Proc. Part. Acc. Conf, Chicago, USA (1989) 
11. van der Meer, S.: Stochastic extraction, a low ripple version of resonant extraction. CERN/PS/AA 78-6 (1978)

12. Grote, H., et al.: MAD-X - an upgrade from MAD8. In: Proceedings of PAC2003. http:// mad.web.cern.ch/mad/ (2003). Accessed 20 Oct 2008.

13. Vector Fields Inc.: Computer program Opera3D. www.vectorfields.com (2008). Accessed 20 Oct 2008 